\title{
Triacylglycerol Lipase Measurement
}

National Cancer Institute

\section{Source}

National Cancer Institute. Triacylglycerol Lipase Measurement. NCI Thesaurus. Code C64807.

A quantitative measurement of the amount of triacylglycerol lipase present in a sample. 